\title{
Cardiovascular Toxicity of Epoetin-Alfa in Patients with Chronic Kidney Disease
}

\author{
Peter A. McCullough ${ }^{\text {a-d }}$ Huiman X. Barnhart ${ }^{f, h}$ Jula K. Inrig ${ }^{i}$ Donal Reddank \\ Shelly Sapp ${ }^{f, h}$ Uptal D. Patel ${ }^{e, f}$ Ajay K. Singh ${ }^{j}$ Lynda A. Szczech $^{e, f}$ \\ Robert M. Califfg \\ ${ }^{a}$ St. John Providence Health System, Warren, Mich., b Providence Hospitals and Medical Centers, Southfield, Mich./ \\ Novi, Mich., 'St. John Hospital and Medical Center, Detroit, Mich., ' St. John Macomb Oakland Center,

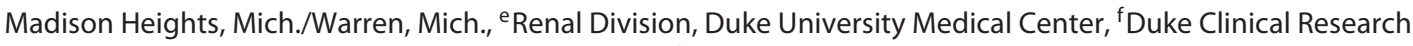 \\ Institute, ${ }^{9}$ Duke Translational Research Institute, and hepartment of Biostatistics and Bioinformatics, \\ Duke University, Durham, N.C., 'University of Texas Southwestern Medical Center, Dallas, Tex., and ${ }^{\mathrm{j}}$ Renal Division,

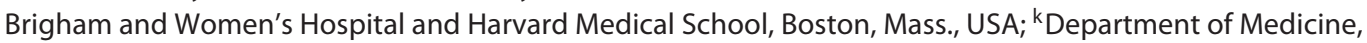 \\ University College Galway, Galway, Ireland
}

\section{Key Words}

Anemia $\cdot$ Cardiovascular disease $\cdot$ Chronic kidney disease .

Epoetin-alfa · Dose · Epidemiology

\begin{abstract}
Background: Recombinant erythropoietin has become a routine component of care of patients with chronic kidney disease reducing the need for blood transfusions but raising the risks for cardiovascular events. We undertook this secondary analysis of subjects enrolled in the Correction of Hemoglobin and Outcomes in Renal Insufficiency (CHOIR) trial to examine the interrelationships between epoetin-alfa maintenance doses utilized and achieved hemoglobin $(\mathrm{Hb})$ irrespective of treatment target and randomized allocation. Methods: We performed a post hoc analysis from the CHOIR trial. Inclusion criteria were $\mathrm{Hb}<11.0 \mathrm{~g} / \mathrm{dl}$ and estimated glomerular filtration rates of $15-50 \mathrm{ml} / \mathrm{min} / 1.73 \mathrm{~m}^{2}$. To be included in the present analysis, subjects needed to be free of the composite event at 4 months, receive epoetin-alfa, and have $\geq 1$ postbaseline $\mathrm{Hb}$ measurement. The mean weekly dose of epoetin-alfa received up to the time of first event or
\end{abstract}

censure was the main exposure variable, while the achieved $\mathrm{Hb}$ at month 4 was the confounder representing the subject's underlying response to treatment. The primary outcome was the composite of death, heart failure hospitalization, stroke, or myocardial infarction. A Cox proportional hazard regression model was used in time-to-event analysis. Results: Among 1,244 subjects with complete data, the average weekly dose of epoetin-alfa ranged 143.3-fold from 133 to 19,106 units/week at the time of first event or censure. Cox proportional hazard analysis found that those in the middle tertile of $\mathrm{Hb}$ achieved ( $>11.5$ to $<12.7 \mathrm{~g} / \mathrm{dl}$ ) and the lowest tertile of epoetin-alfa dose exposure level $(<5,164$ units/ week) had the lowest risk. Irrespective of $\mathrm{Hb}$ achieved, the relative risk in the highest tertile (>10,095 units/week) of epoetin-alfa dose exposure level was significantly escalated (hazard ratios ranged from 2.536 to $3.572, p<0.05$, when compared to the group of middle $\mathrm{Hb}$ tertile and lowered dose tertile). In a multivariable model that adjusted for achieved $\mathrm{Hb}$, albumin, cholesterol, age, prior heart failure, prior stroke, prior deep venous thrombosis, atrial fibrillation or malignancy, the average weekly dose had a significant $(p=0.005)$ relative risk of 1.067 per 1,000 units of epoetin-

\section{KARGER}

E-Mail karger@karger.com

www.karger.com/ajn
(C) 2013 S. Karger AG, Basel

0250-8095/13/0376-0549\$38.00/0
Peter A. McCullough, MD, MPH, FACC, FACP, FAHA, FCCP

St. John Providence Health System

47601 Grand River Avenue, Suite B-125

Novi, MI 48374 (USA)

E-Mail peteramccullough@gmail.com 
alfa for the primary end point. Conclusions: In the CHOIR trial, average epoetin-alfa doses $>10,095$ units/week were associated with increased risks for cardiovascular events irrespective of the $\mathrm{Hb}$ achieved within the first 4 months of treatment. These data suggest the weekly epoetin-alfa dose and not the $\mathrm{Hb}$ achieved was a principal determinant in the primary outcome observed implicating a cardiovascular toxicity of this erythrocyte-stimulating agent.

Copyright $\odot 2013$ S. Karger AG, Basel

\section{Introduction}

Anemia is a common development in patients with advanced chronic illness including heart and kidney disease. Common causes for reduced hemoglobin $(\mathrm{Hb})$ concentration in cardiorenal disease include reduced production of erythrocytes due to impairment in iron utilization, relative erythropoietin deficiency, and resistance to the actions of erythropoietin. Anemia has been consistently associated with the progression of chronic kidney disease and increased rates of cardiovascular disease events including myocardial infarction, stroke, and heart failure. Recombinant erythropoietin has become a routine component of care of patients with chronic kidney disease and end-stage renal disease reducing the need for blood transfusions $[1,2]$. One randomized trial of supplemental erythrocyte-stimulating agents (ESA) in endstage renal disease and 3 randomized trials in predialysis chronic kidney disease patients either failed to demonstrate a benefit of using ESAs to achieve higher $\mathrm{Hb}$ targets, or found an increased risk of the cardiovascular composite outcome. Specifically, an increased risk for cardiovascular hospitalization was seen in the Correction of Hemoglobin and Outcomes in Renal Insufficiency (CHOIR) trial while an elevated risk of stroke was observed in the Trial to Reduce Cardiovascular Events with Aranesp Therapy (TREAT) [3-7]. In the CHOIR study, among those with heart failure or diabetes at baseline, there was no increased risk of cardiovascular outcomes with higher $\mathrm{Hb}$ targets, suggesting a lack of internal consistency with respect to the $\mathrm{Hb}$ achieved and cardiovascular outcome [8]. Additionally, in the TREAT, cardiovascular risk was most strongly predicted by age, history of heart failure, C-reactive protein, urinary protein/creatinine ratio, abnormal electrocardiogram, serum $\mathrm{N}$-terminal pro-B-type natriuretic peptide, and troponin $\mathrm{T}$, but not the achieved $\mathrm{Hb}$ [9].

Three major explanations have emerged regarding ESA use and risks of cardiovascular events. The first is that elevation in $\mathrm{Hb}$ as a result of ESA therapy, through increasing blood volume or viscosity or other pathways, works to promote cardiovascular disease. It is possible that some physicians in the research and practice community have subscribed to the concept that elevation of $\mathrm{Hb}$ is the proximate cause of increased cardiovascular risk $[10,11]$. The preponderance of evidence to date, however, indicates that elevation of $\mathrm{Hb}$ into the normal range occurring spontaneously is not pathogenic, but is associated with reduced rates of cardiovascular events [12]. The second is that ESA resistance reflects a state of advanced illness which confounds the relationship between cardiovascular outcomes and exogenous ESA administration, thus ESA usage itself is neutral on the cardiovascular system $[13,14]$. While ESA resistance has been associated with generalized illness, androgen deficiency, infection and inflammation, increased cytokine signaling, secondary hyperparathyroidism, use of drugs that antagonize the renin-angiotensin system, and acquired defects in iron transport, there are however no plausible explanations of how resistance to ESA could cause or promote atherosclerosis, myocardial disease, or other forms of cardiovascular disease $[15,16]$. The third is that ESA therapy, in supraphysiologic doses, has a cardiovascular toxicity related to the average, median, or cumulative exposure over time. Biologic explanations for ESA causing or worsening cardiovascular disease include increased sensitivity of $\alpha$-adrenergic receptors, elevations in blood pressure, and increased thrombogenicity with supraphysiologic exposure to this protein $[17,18]$. Thus, if the third explanation is correct, then in those with the lowest ESA exposure and the highest $\mathrm{Hb}$ achieved, there would be a lower cardiovascular risk compared to those with higher ESA at any achieved $\mathrm{Hb}$ value as suggested in the Normal Hematocrit Study in patients with end-stage renal disease [19].

This secondary analysis of subjects with chronic kidney disease enrolled in the CHOIR trial was undertaken to examine the interrelationships between epoetin-alfa doses utilized and achieved $\mathrm{Hb}$ irrespective of treatment target and randomized allocation. A previous analysis from the CHOIR study had demonstrated that participants receiving 'high-dose' ESA therapy ( $\geq 20,000$ units/ week) by 4 and 9 months had increased cardiovascular events over the course of the trial irrespective of the treatment arm (HR $=1.71$ at 4 months, $\mathrm{p}=0.003 ; \mathrm{HR}=1.54$ at 9 months, $\mathrm{p}=0.05$ ) [20]. These associations remained statistically significant when adjusting for ESA resistance and other confounders. This prior analysis, however, did not examine what happened to the dose and exposure to
McCullough/Barnhart/Inrig/Reddan/ Sapp/Patel/Singh/Szczech/Califf 
epoetin-alfa after 9 months of treatment in the trial. We sought to extend these observations and critically evaluate the entirety of drug exposure up to the time of the first event or censure in participants with available information. We hypothesized that higher ESA dose exposure would be associated with increased CV morbidity and mortality among participants in the CHOIR trial.

\section{Methods}

Study Subjects

The CHOIR was a randomized trial comparing the effect of treatment with epoetin-alfa to one of two $\mathrm{Hb}$ targets on the composite end point of death, heart failure, stroke, and myocardial infarction in chronic kidney disease patients. Methods, baseline characteristics, and results of the CHOIR trial have been previously reported [21]. Inclusion criteria were $\mathrm{Hb}<11.0 \mathrm{~g} / \mathrm{dl}$ and an estimated glomerular filtration rate of $15-50 \mathrm{ml} / \mathrm{min} / 1.73 \mathrm{~m}^{2}$ [22].

Of the 1,432 subjects randomized, 25 were excluded because they had not received epoetin-alfa or obtained postbaseline $\mathrm{Hb}$ measurement. At 4 months, 147 subjects were excluded because they had events or terminated the study prior to this landmark, and 36 were excluded because of incomplete $\mathrm{Hb}$ or ESA dose data. This approach yielded a total of 1,224 subjects for analysis.

\section{Measurements}

Subjects in the CHOIR trial were randomized to $\mathrm{Hb}$ targets of 11.3 or $13.5 \mathrm{~g} / \mathrm{dl}$ utilizing different dosing algorithms and were administered weekly or biweekly epoetin-alfa subcutaneously as previously published. Information on ESA dose and $\mathrm{Hb}$ were collected at least biweekly. We chose the achieved $\mathrm{Hb}$ by month 4 to represent the subject's underlying resistance status in response to ESA treatment as done previously in a CHOIR landmark analysis [8]. We chose $\mathrm{Hb}$ tertile as the analytic framework for the results tables since it objectively divided the study population into three groups and is a familiar treatment target to clinicians, guideline developers, and regulatory agencies. In order to determine whether the overall dose exposure, not just the exposure up to month 4, is associated with the primary outcome regardless of resistance status, we chose the average weekly dose (calculated with all recorded doses over the total observation time, i.e. up to time of first event or censure) as the primary exposure variable.

\section{Statistical Analysis}

Patient characteristics were described by tertiles of achieved $\mathrm{Hb}$ at month 4 (table 1). Continuous data were summarized by mean (with standard deviation) or median, and categorical data were summarized by counts and proportions. The Kruskal-Wallis and $\chi^{2}$ tests were used for continuous and categorical variables, respectively, in making comparisons between the tertiles of achieved $\mathrm{Hb}$ at months. Cox proportional hazard analyses were performed to examine associations between achieved $\mathrm{Hb}$ at 4 months, average weekly epoetin-alfa dose, and the primary outcome of the composite of death, heart failure hospitalization, stroke, or myocardial infarction reported as Kaplan-Meier event rates at 3 years with logrank $\mathrm{p}$ values for differences between $\mathrm{Hb}$ groups. The achieved $\mathrm{Hb}$ at month 4 and average weekly ESA dose as continuous variables were then used in a model-building process shown as models 1-3 in table 2 while adjusting for known significant baseline variables to arrive at the final model $4[8,20]$. A p value less than 0.05 was considered statistically significant. All statistical analyses were performed using SAS (version 8.2, SAS Institute, Cary, N.C., USA).

\section{Results}

Of the available 1,260 subjects for the 4-month (study days $92-122$, inclusive) landmark analysis population, 29 did not have $\mathrm{Hb}$ reported, 3 had no dosing data on or after day 92 and 4 were missing both $\mathrm{Hb}$ values and ESA doses. Therefore, 1,224 patients were included in the analyses (table 1). Those in the highest tertile of $\mathrm{Hb}$ achieved were more likely to be Hispanic and not smoking (table 1). Of note, those with the highest $\mathrm{Hb}$ achieved were more likely to have heart failure at baseline, $\mathrm{p}=$ 0.034 . Hb at baseline and after epoetin-alfa 10,000 units subcutaneously once per week for 3 consecutive weeks regardless of weight or baseline $\mathrm{Hb}$ were within a tight range of 9.9-10.3, and 10.3-10.9 g/dl (lowest to highest tertile), despite being statistically significant $(\mathrm{p}<0.001)$.

The average weekly dose of epoetin-alfa ranged 143.3fold from 133 to 19,106 units/week at the time of first event or censure. Primary end points occurred in $154 / 1,224$ (12.6\%) subjects. The medians (with interquartile ranges) of epoetin-alfa (averaged over the first 4 months) and the weekly dose of epoetin-alfa received at the time of first primary event compared to those without events at censure were: $8,000.0(2,500-16,000)$ versus $5,200.0(1,500-12,000)$, and $8,945.3(5,829-12,807)$ versus $7,093.9(3,853-11,200)$ units, $\mathrm{p}=0.001$, and $\mathrm{p}<0.001$, respectively. The cumulative dose of epoetin-alfa received up to the time of first event or censure ranged from 40,000 to $2,262,000$ units (56.55-fold variation). Figure 1 provides a descriptive risk profile (based on hazard ratios) of 9 groups based on the combinations of the achieved $\mathrm{Hb}$ tertiles and average weekly ESA dose tertiles for the primary outcome where the reference group is the lowest risk group: middle tertile of achieved $\mathrm{Hb}(11.5<\mathrm{Hb}<12.7$ $\mathrm{g} / \mathrm{dl})$ and lowest tertile of average weekly ESA dose $(<5,164$ units/week). Model 1 in table 2 demonstrates that there is an overall significant difference across the 9 groups ( $\mathrm{p}=$ 0.0074 ). The corresponding $p$ values for the hazard ratios for each of the 8 groups as compared to the lowest risk group are also shown in table 2 model 1 . Irrespective of $\mathrm{Hb}$ achieved, the relative risks in the highest tertile (>10,095 units/week corresponding to a cumulative median dose of 166,000 ) of epoetin-alfa dose exposure level 
Table 1. Baseline characteristics of subjects and end points between randomization and termination

\begin{tabular}{|c|c|c|c|c|c|}
\hline & \multicolumn{3}{|c|}{ Average $\mathrm{Hb}$ group in month 4 tertile } & \multirow{2}{*}{$\begin{array}{l}\text { All patients } \\
(\mathrm{n}=1,224)\end{array}$} & \multirow[t]{2}{*}{$\mathrm{p}$ value } \\
\hline & $\begin{array}{l}\text { lowest } \\
(\leq 11.5 \mathrm{~g} / \mathrm{dl}) \\
(\mathrm{n}=415)\end{array}$ & $\begin{array}{l}\text { middle } \\
(>11.5 \text { to }<12.7 \mathrm{~g} / \mathrm{dl}) \\
(\mathrm{n}=399)\end{array}$ & $\begin{array}{l}\text { highest } \\
(\geq 12.7 \mathrm{~g} / \mathrm{dl}) \\
(\mathrm{n}=410)\end{array}$ & & \\
\hline \multirow[t]{2}{*}{ Age, years } & $\mathrm{n}=415$ & $\mathrm{n}=399$ & $\mathrm{n}=410$ & $\mathrm{n}=1,224$ & \\
\hline & $66.2 \pm 13.71$ & $66.3 \pm 13.78$ & $66.2 \pm 13.69$ & $66.2 \pm 13.72$ & 0.967 \\
\hline Female gender & $237 / 415(57.1)$ & $230 / 399(57.6)$ & $214 / 410(52.2)$ & $681 / 1,224(55.6)$ & 0.225 \\
\hline Race, $\mathrm{n}$ & $\mathrm{n}=415$ & $\mathrm{n}=397$ & $\mathrm{n}=410$ & $\mathrm{n}=1,222$ & 0.359 \\
\hline White & 257 (61.9) & $243(61.2)$ & $264(64.4)$ & $764(62.5)$ & \\
\hline Black & $132(31.8)$ & $117(29.5)$ & $104(25.4)$ & $353(28.9)$ & \\
\hline American Indian or Alaskan native & $2(0.5)$ & $1(0.3)$ & $1(0.2)$ & $4(0.3)$ & \\
\hline Asian or Pacific Islander & $8(1.9)$ & $13(3.3)$ & $14(3.4)$ & $35(2.9)$ & \\
\hline Other & $16(3.9)$ & $23(5.8)$ & $27(6.6)$ & $66(5.4)$ & \\
\hline Hispanic ethnicity & $43 / 415(10.4)$ & $45 / 396(11.4)$ & $67 / 409(16.4)$ & $155 / 1,220(12.7)$ & $0.021^{*}$ \\
\hline Cigarette smoking, $\mathrm{n}$ & $\mathrm{n}=415$ & $\mathrm{n}=397$ & $\mathrm{n}=409$ & $\mathrm{n}=1,221$ & $0.049^{*}$ \\
\hline Never & $234(56.4)$ & $227(57.2)$ & $216(52.8)$ & $677(55.4)$ & \\
\hline Past & $147(35.4)$ & $150(37.8)$ & $175(42.8)$ & $472(38.7)$ & \\
\hline Current & $34(8.2)$ & $20(5.0)$ & $18(4.4)$ & $72(5.9)$ & \\
\hline \multicolumn{6}{|l|}{$\overline{\text { Past/current cigarette smoking }}$} \\
\hline Current smoker (vs. never/past smoker) & $34 / 415(8.2)$ & $20 / 397(5.0)$ & $18 / 409(4.4)$ & $72 / 1,221(5.9)$ & $0.047^{*}$ \\
\hline Primary etiology, $\mathrm{n}$ & $\mathrm{n}=412$ & $\mathrm{n}=396$ & $\mathrm{n}=406$ & $\mathrm{n}=1,214$ & 0.379 \\
\hline Diabetes & $206(50.0)$ & $181(45.7)$ & $194(47.8)$ & $581(47.9)$ & \\
\hline Hypertension & $109(26.5)$ & $130(32.8)$ & $116(28.6)$ & $355(29.2)$ & \\
\hline Other & $97(23.5)$ & $85(21.5)$ & $96(23.6)$ & $278(22.9)$ & \\
\hline Prior diabetes or diabetes etiology & $274 / 415(66.0)$ & $239 / 397(60.2)$ & $244 / 408(59.8)$ & $757 / 1,220(62.0)$ & 0.120 \\
\hline \multicolumn{6}{|l|}{ Cardiovascular history, $\mathrm{n}$} \\
\hline Hypertension & $375 / 395(94.9)$ & $351 / 382(91.9)$ & $378 / 391(96.7)$ & $1,104 / 1,168(94.5)$ & $0.013^{*}$ \\
\hline MI & $61 / 394(15.5)$ & $55 / 383(14.4)$ & $57 / 389(14.7)$ & $173 / 1,166(14.8)$ & 0.901 \\
\hline CABG & $49 / 394(12.4)$ & $54 / 383(14.1)$ & $78 / 390(20.0)$ & $181 / 1,167(15.5)$ & $0.009 *$ \\
\hline PCI & $52 / 394(13.2)$ & $35 / 383(9.1)$ & $32 / 389(8.2)$ & $119 / 1,166(10.2)$ & $0.050^{*}$ \\
\hline $\mathrm{CHF}$ & $96 / 394(24.4)$ & $80 / 382(20.9)$ & $74 / 389(19.0)$ & $250 / 1,165(21.5)$ & 0.182 \\
\hline Atrial fibrillation/flutter & 43/394 (10.9) & $29 / 382(7.6)$ & $23 / 389(5.9)$ & $95 / 1,165(8.2)$ & $0.034^{*}$ \\
\hline CVA & $36 / 393(9.2)$ & $39 / 382(10.2)$ & $34 / 390(8.7)$ & $109 / 1,165(9.4)$ & 0.766 \\
\hline Lower extremity amputation & $9 / 393(2.3)$ & $14 / 382(3.7)$ & $11 / 390(2.8)$ & $34 / 1,165(2.9)$ & 0.519 \\
\hline MI, CABG or PCI & $140 / 394(35.5)$ & $130 / 381(34.1)$ & $130 / 390(33.3)$ & $400 / 1,165(34.3)$ & 0.806 \\
\hline \multirow[t]{2}{*}{ Baseline mean body mass index } & $\mathrm{n}=391$ & $\mathrm{n}=383$ & $\mathrm{n}=399$ & $\mathrm{n}=1,173$ & \\
\hline & $30.7 \pm 8.14$ & $30.6 \pm 7.61$ & $30.1 \pm 7.28$ & $30.5 \pm 7.68$ & 0.689 \\
\hline \multirow{2}{*}{$\begin{array}{l}\text { Baseline mean GFR, } \\
\mathrm{ml} / \mathrm{min} / 1.73 \mathrm{~m}^{2}\end{array}$} & $\mathrm{n}=415$ & $\mathrm{n}=399$ & $\mathrm{n}=409$ & $\mathrm{n}=1,223$ & \\
\hline & $27.4 \pm 9.14$ & $27.4 \pm 9.00$ & $27.2 \pm 8.59$ & $27.3 \pm 8.91$ & 0.987 \\
\hline \multirow{2}{*}{$\begin{array}{l}\text { Baseline mean creatinine, } \\
\mathrm{mg} / \mathrm{dl}\end{array}$} & $\mathrm{n}=415$ & $\mathrm{n}=399$ & $\mathrm{n}=409$ & $\mathrm{n}=1,223$ & \\
\hline & $2.4 \pm 0.76$ & $2.4 \pm 0.76$ & $2.4 \pm 0.76$ & $2.4 \pm 0.76$ & 0.950 \\
\hline \multirow{2}{*}{$\begin{array}{l}\text { Baseline mean } \mathrm{Hb}, \\
\mathrm{g} / \mathrm{dl}\end{array}$} & $\mathrm{n}=411$ & $\mathrm{n}=397$ & $\mathrm{n}=408$ & $\mathrm{n}=1,216$ & \\
\hline & $9.9 \pm 0.87$ & $10.2 \pm 0.84$ & $10.3 \pm 0.76$ & $10.1 \pm 0.84$ & $<0.001^{*}$ \\
\hline \multirow{2}{*}{$\begin{array}{l}\text { Week } 3 \text { mean } \mathrm{Hb} \text {, } \\
\mathrm{g} / \mathrm{dl}\end{array}$} & $\mathrm{n}=365$ & $\mathrm{n}=360$ & $\mathrm{n}=371$ & $\mathrm{n}=1,096$ & \\
\hline & $10.3 \pm 0.91$ & $10.7 \pm 0.91$ & $10.9 \pm 0.89$ & $10.6 \pm 0.93$ & $<0.001^{*}$ \\
\hline \multirow{2}{*}{$\begin{array}{l}\text { Baseline mean albumin, } \\
\mathrm{g} / \mathrm{dl}\end{array}$} & $\mathrm{n}=415$ & $\mathrm{n}=399$ & $\mathrm{n}=409$ & $\mathrm{n}=1,223$ & \\
\hline & $3.7 \pm 0.47$ & $3.8 \pm 0.50$ & $3.8 \pm 0.48$ & $3.8 \pm 0.48$ & $0.020^{*}$ \\
\hline \multirow{2}{*}{$\begin{array}{l}\text { Baseline mean phosphorus, } \\
\mathrm{mg} / \mathrm{dl}\end{array}$} & $\mathrm{n}=415$ & $\mathrm{n}=399$ & $\mathrm{n}=408$ & $\mathrm{n}=1,222$ & \\
\hline & $4.1 \pm 0.76$ & $4.0 \pm 0.70$ & $4.1 \pm 0.75$ & $4.1 \pm 0.74$ & 0.493 \\
\hline \multirow{2}{*}{$\begin{array}{l}\text { Baseline mean cholesterol, } \\
\mathrm{mg} / \mathrm{dl}\end{array}$} & $\mathrm{n}=415$ & $\mathrm{n}=399$ & $\mathrm{n}=409$ & $\mathrm{n}=1,223$ & \\
\hline & $182.3 \pm 48.65$ & $187.8 \pm 52.07$ & $182.7 \pm 45.29$ & $184.2 \pm 48.75$ & 0.486 \\
\hline \multirow{2}{*}{$\begin{array}{l}\text { Baseline mean urine total protein/ } \\
\text { creatinine ratio }\end{array}$} & $\mathrm{n}=396$ & $\mathrm{n}=392$ & $\mathrm{n}=393$ & $\mathrm{n}=1,181$ & \\
\hline & $1.5 \pm 2.09$ & $1.4 \pm 2.17$ & $1.5 \pm 2.08$ & $1.4 \pm 2.11$ & 0.804 \\
\hline \multirow{2}{*}{$\begin{array}{l}\text { Baseline mean ferritin, } \\
\mathrm{ng} / \mathrm{ml}\end{array}$} & $\mathrm{n}=413$ & $\mathrm{n}=397$ & $\mathrm{n}=409$ & $\mathrm{n}=1,219$ & \\
\hline & $155.7 \pm 159.96$ & $181.9 \pm 168.03$ & $180.7 \pm 160.80$ & $172.6 \pm 163.22$ & $0.002^{*}$ \\
\hline \multirow[t]{2}{*}{ Baseline mean TSAT, \% } & $\mathrm{n}=415$ & $\mathrm{n}=399$ & $\mathrm{n}=408$ & $\mathrm{n}=1,222$ & \\
\hline & $23.7 \pm 9.74$ & $25.4 \pm 11.00$ & $25.9 \pm 11.73$ & $25.0 \pm 10.88$ & $0.037^{*}$ \\
\hline Baseline TSAT <20\% & $159 / 415(38.3)$ & $132 / 399(33.1)$ & $132 / 408(32.4)$ & $423 / 1,222(34.6)$ & 0.146 \\
\hline
\end{tabular}


Table 1 (continued)

\begin{tabular}{|c|c|c|c|c|c|}
\hline & \multicolumn{3}{|c|}{ Average Hb group in month 4 tertile } & $\begin{array}{l}\text { All patients } \\
(\mathrm{n}=1,224)\end{array}$ & $\mathrm{p}$ value \\
\hline Baseline medications, $\mathrm{n}$ & $\mathrm{n}=413$ & $\mathrm{n}=394$ & $\mathrm{n}=407$ & $\mathrm{n}=1,214$ & \\
\hline ACE inhibitor and/or ARB & $304(73.6)$ & $305(77.4)$ & $307(75.4)$ & $916(75.5)$ & 0.455 \\
\hline$\beta$-Blocker & $200(48.4)$ & $181(45.9)$ & $183(45.0)$ & $564(46.5)$ & 0.591 \\
\hline Intravenous & $10(2.4)$ & $9(2.3)$ & $9(2.2)$ & $28(2.3)$ & 0.980 \\
\hline Oral & $110(26.6)$ & $98(24.9)$ & $113(27.8)$ & $321(26.4)$ & 0.647 \\
\hline Unknown route & $9(2.2)$ & $6(1.5)$ & $15(3.7)$ & $30(2.5)$ & 0.128 \\
\hline \multicolumn{5}{|l|}{ Average weekly epoetin-alfa dose } & $0.002 *$ \\
\hline Mean \pm SD & $8,330.7 \pm 7,169.33$ & $6,732.6 \pm 6,713.44$ & $7,396.0 \pm 6,225.95$ & $7,496.6 \pm 6,742.36$ & \\
\hline Median & 6,000 & 4,500 & 6,000 & 5,600 & \\
\hline Mean \pm SD & $8,155.4 \pm 4,947.51$ & $7,163.6 \pm 4,885.33$ & $8,694.4 \pm 3,890.76$ & $8,012.6 \pm 4,638.25$ & \\
\hline Median & $6,813.2$ & $6,043.6$ & $8,571.9$ & $7,374.8$ & \\
\hline 25th-75th percentile & $3,885.1-12,441.9$ & $3,043.5-10,926.5$ & $6,066.3-11,331.0$ & $3,952.9-11,489.5$ & \\
\hline Min.-max. & $133.3-18,657.1$ & $375.0-19,105.7$ & $315.8-18,592.6$ & $133.3-19,105.7$ & \\
\hline \multicolumn{5}{|c|}{ Cumulative epoetin-alfa dose through month 4} & $<0.001^{*}$ \\
\hline Mean \pm SD & $150,549.4 \pm 86,315.48$ & $143,373.4 \pm 78,318.48$ & $168,305.9 \pm 64,936.21$ & $154,158.0 \pm 77,703.17$ & \\
\hline Median & 124,000 & 120,000 & 166,000 & 143,000 & \\
\hline 25th-75th percentile & $80,000-227,000$ & $78,000-200,000$ & $117,000-213,000$ & $90,000-214,000$ & \\
\hline Min.-max. & $0-330,000$ & $0-330,000$ & $30,000-329,000$ & $0-330,000$ & \\
\hline \multicolumn{6}{|l|}{ Cumulative epoetin-alfa dose through day } \\
\hline of the 1st event, units/week & $\mathrm{n}=58$ & $\mathrm{n}=43$ & $\mathrm{n}=53$ & $\mathrm{n}=154$ & 0.980 \\
\hline Mean \pm SD & $553,862.1-447,079.39$ & $506,897.7-372,718.72$ & $514,037.7-340,344.89$ & $527,042.9-390,402.01$ & \\
\hline Mean \pm SD & $10.78 \pm 0.669$ & $12.05 \pm 0.313$ & $13.57 \pm 0.614$ & $12.13 \pm 1.272$ & \\
\hline Min.-max. & $6.95-11.50$ & $11.53-12.67$ & $12.70-15.60$ & $6.95-15.60$ & \\
\hline \multicolumn{6}{|c|}{ End points between randomization and termination } \\
\hline MI & $11 / 415(5.9)$ & $9 / 399(2.9)$ & $11 / 410(4.4)$ & $31 / 1,224(4.6)$ & 0.932 \\
\hline Stroke & $7 / 415(6.4)$ & $5 / 399(1.4)$ & $6 / 410(4.4)$ & $18 / 1,224(4.3)$ & 0.923 \\
\hline Heart failure hospitalization & $22 / 415(7.9)$ & $20 / 399(10.0)$ & $23 / 410(8.3)$ & $65 / 1,224(8.7)$ & 0.944 \\
\hline Death & $22 / 415(8.8)$ & $20 / 399(14.6)$ & $23 / 410(11.4)$ & $65 / 1,224(11.5)$ & 0.932 \\
\hline Primary composite end point & $58 / 415(25.5)$ & $43 / 399(21.5)$ & $53 / 410(23.1)$ & $154 / 1,224(23.6)$ & 0.481 \\
\hline \multicolumn{6}{|c|}{$\begin{array}{l}{ }^{*} \mathrm{p}<0.05 \text { : threshold for statistically significant values. Figures in parentheses indicate percentages. } \mathrm{MI}=\text { Myocardial infarction; } \mathrm{CABG}=\text { coronary artery } \\
\text { bypass graft; } \mathrm{PCI}=\text { percutaneous coronary intervention; } \mathrm{CHF}=\text { congestive heart failure; } \mathrm{CVA}=\text { cerebrovascular accident; } \mathrm{GFR}=\text { glomerular filtration rate; } \\
\text { TSAT = transferrin saturation; } \mathrm{ACE}=\text { angiotensin-converting enzyme; } \mathrm{ARB}=\text { angiotensin receptor blocker; HMG-CoA }=3 \text {-hydroxy-3-methylglutaryl co- } \\
\text { enzyme A. Test statistics: continuous variables }- \text { Kruskal-Wallis test; binary/categorical variables }- \text { Pearson's } \chi^{2} \text { test; end points between randomization and } \\
\text { termination - log-rank test. Continuous variables are reported as means } \pm \mathrm{SD} \text { unless noted otherwise; number and percentages are reported for binary/cat- } \\
\text { egorical variables; number of events and Kaplan-Meier event rates at } 36 \text { months are reported for end points between randomization and termination. As for } \\
\text { the primary composite end point of death, heart failure hospitalization, stroke or myocardial infarction, patients can experience more than one end point. } \\
\text { For the primary composite end point, time to the first end point is used in the analysis. }\end{array}$} \\
\hline
\end{tabular}


Table 2. Cox proportional hazard models for the primary composite end point of death, heart failure hospitalization, stroke or myocardial infarction, according to the Hb achieved at 4 months and the average weekly epoetinalfa dose exposure prior to the first event or censure

\begin{tabular}{|c|c|c|}
\hline Variable & HR & $\mathrm{p}$ value \\
\hline \multicolumn{3}{|l|}{ Model 1} \\
\hline \multicolumn{3}{|l|}{$\begin{array}{l}\text { Combinations of achieved } \mathrm{Hb} \text { tertile in month } 4 \text { and average weekly } \\
\text { epoetin-alfa dose tertile prior to first event or censure }\end{array}$} \\
\hline Lowest, lowest & $1.363(0.618-3.006)$ & 0.443 \\
\hline Lowest, middle & $2.679(1.274-5.629)$ & 0.009 \\
\hline Lowest, highest & $3.572(1.757-7.260)$ & $<0.001$ \\
\hline Middle, lowest (reference group) & $1.0(\mathrm{NA})$ & NA \\
\hline Middle, middle & $2.124(0.951-4.745)$ & 0.066 \\
\hline Middle, highest & $3.304(1.572-6.945)$ & 0.002 \\
\hline Highest, lowest & $1.456(0.565-3.758)$ & 0.437 \\
\hline Highest, middle & $2.383(1.167-4.866)$ & 0.017 \\
\hline Highest, highest & $2.536(1.230-5.229)$ & 0.012 \\
\hline \multicolumn{3}{|l|}{ Model 2} \\
\hline Average $\mathrm{Hb}$ in month 4 & $1.181(0.878-1.589)$ & 0.271 \\
\hline Average weekly epoetin-alfa dose (per 1,000 units) prior to first event or censure & $1.360(0.982-1.884)$ & 0.065 \\
\hline Interaction between average $\mathrm{Hb}$ and average weekly epoetin-alfa dose & $0.980(0.954-1.008)$ & 0.154 \\
\hline \multicolumn{3}{|l|}{ Model 3} \\
\hline Average $\mathrm{Hb}$ in month 4 & $0.971(0.858-1.098)$ & 0.635 \\
\hline $\begin{array}{l}\text { Average weekly epoetin-alfa dose (per 1,000 units) prior to the first event or } \\
\text { censure }\end{array}$ & $1.074(1.040-1.109)$ & $<0.001$ \\
\hline \multicolumn{3}{|l|}{ Model 4} \\
\hline Average $\mathrm{Hb}$ in month 4 & $1.020(0.870-1.197)$ & 0.805 \\
\hline Average weekly epoetin-alfa dose to day of 1 st event or censure (per 1,000 units) & $1.067(1.020-1.116)$ & 0.005 \\
\hline $\mathrm{Hb} 13.5 \mathrm{~g} / \mathrm{dl}$ (vs. $11.3 \mathrm{~g} / \mathrm{dl})$ (actual) & $0.965(0.585-1.591)$ & 0.889 \\
\hline Baseline albumin & $0.714(0.503-1.015)$ & 0.060 \\
\hline \multicolumn{3}{|l|}{ Baseline cholesterol linear splines (per $10 \mathrm{mg} / \mathrm{dl}$ ) } \\
\hline$<240 \mathrm{mg} / \mathrm{dl}$ & $0.974(0.933-1.017)$ & 0.238 \\
\hline$\geq 240 \mathrm{mg} / \mathrm{dl}$ & $1.089(1.010-1.174)$ & 0.027 \\
\hline \multicolumn{3}{|l|}{ Age linear splines (per 5 years) } \\
\hline$<55$ years & $1.481(1.031-2.128)$ & 0.033 \\
\hline$\geq 55$ to $<75$ years & $0.966(0.830-1.125)$ & 0.660 \\
\hline$\geq 75$ years & $1.442(1.163-1.789)$ & $<0.001$ \\
\hline Prior CHF composite & $2.466(1.765-3.445)$ & $<0.001$ \\
\hline Prior stroke or transient ischemic attack & $1.309(0.869-1.971)$ & 0.198 \\
\hline Prior deep venous thrombosis & $2.540(1.350-4.780)$ & 0.004 \\
\hline History of solid organ malignancy & $0.599(0.359-1.002)$ & 0.051 \\
\hline Prior atrial fibrillation/flutter & $1.835(1.182-2.848)$ & 0.007 \\
\hline
\end{tabular}

$\mathrm{HR}=$ Hazard ratio; 95\% confidence intervals in parentheses; NA = not assessed. Reference group for model 1: average $\mathrm{Hb}$ middle tertile, average epoetin-alfa dose middle tertile. $\mathrm{CHF}=$ Congestive heart failure; prior $\mathrm{CHF}$ composite: prior CHF, cardiomyopathy, left ventricular dysfunction, or right ventricular dysfunction.

were significantly escalated (hazard ratios ranged from 2.536 to $3.572 ; \mathrm{p}<0.05$ when compared to the lowest risk group of middle $\mathrm{Hb}$ tertile and lowest dose tertile). The relative risk in the middle tertile ( $>5,164$ to $<10,095$ units/ week) as compared to the lowest tertile was significantly or almost significantly increased as well, irrespective of
$\mathrm{Hb}$ achieved. When the highest risk group (the lowest tertile of the achieved $\mathrm{Hb}$ and the highest tertile of the average weekly ESA dose) was compared to the combined remaining 8 groups, the log-rank p value was 0.0085 as shown with the Kaplan-Meier survival curves in figure 2. There was no significant interaction $(\mathrm{p}=0.83)$ between 


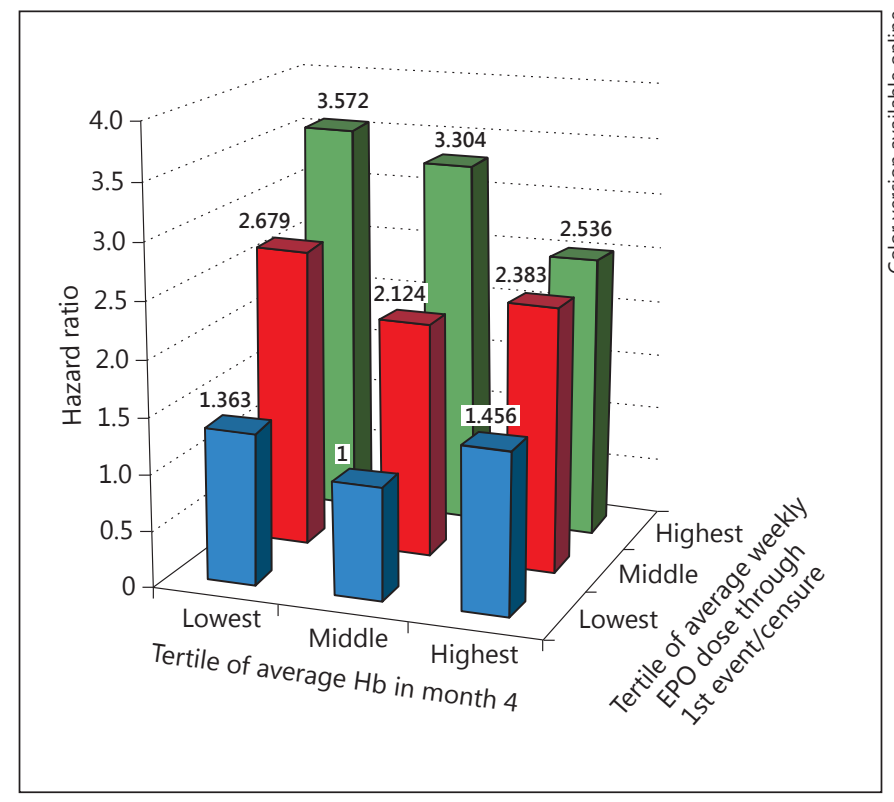

Fig. 1. Cox proportional hazards for the primary composite end point of death, heart failure hospitalization, stroke, or myocardial infarction, according to the tertile of $\mathrm{Hb}$ achieved at 4 months and the long-term maintenance epoetin-alfa (EPO) dose received. The middle tertile is set as referent. The $\mathrm{x}$-axis represents average $\mathrm{Hb}$ in month 4, and the z-axis represents the average weekly dose of EPO received prior to the first event or time of censure. Average $\mathrm{Hb}$ tertiles were $(\mathrm{g} / \mathrm{dl})$ : lowest $\leq 11.5$, middle $>11.5$ to $<12.7$, highest $\geq 12.7$. Average weekly EPO dose tertiles prior to first event were (1,000 units): lowest $<5,164$, middle 5,164-10,095, highest $>10,095$.

the achieved $\mathrm{Hb}$ tertiles and the average weekly ESA dose tertiles. The model with main effects only of achieved $\mathrm{Hb}$ tertiles and the average weekly ESA dose tertiles indicated that there was no significant impact of achieved $\mathrm{Hb}$ tertiles $(\mathrm{p}=0.51)$; however, there was a significant effect of average weekly ESA dose $(p=0.0001)$ on the primary outcome. The same findings are also shown when achieved $\mathrm{Hb}$ and average weekly dose, and the interaction between the two, are treated as continuous variables in model 2 of table 2. Model 3 demonstrates that when the interaction between average $\mathrm{Hb}$ and average weekly epoetin-alfa dose is removed, the relationship between drug dose and outcome remains significant. In the fully adjusted model 4 , the average weekly dose had a relative risk of 1.067 per 1,000 units of epoetin-alfa $(\mathrm{p}=0.005)$ after adjustment for achieved $\mathrm{Hb}$, albumin, cholesterol, age, prior heart failure, prior stroke, prior deep venous thrombosis, atrial fibrillation, or malignancy. Of note, baseline estimated glomerular filtration rate was considered as a potential co-

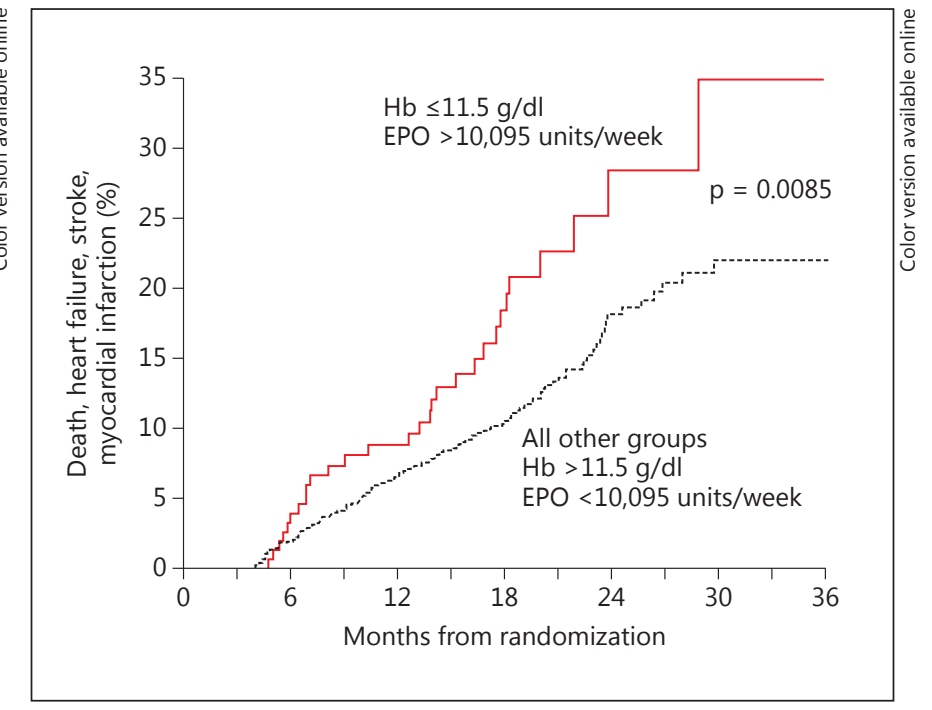

Fig. 2. Kaplan-Meier survival curves for the lowest tertile of the average $\mathrm{Hb}$ achieved in month 4 for patients $(\mathrm{n}=1,224)$ who went on to receive the highest average weekly epoetin-alfa (EPO) dose up to the time of the first event or censure $(\mathrm{Hb} \leq 11.5 \mathrm{~g} / \mathrm{dl}$ with EPO $>10,095$ units/week) compared to the remaining 8 tertiles by tertile groups ( $\mathrm{Hb}>11.5 \mathrm{~g} / \mathrm{dl}$ with $\mathrm{EPO}<10,095$ units/week), log-rank $\mathrm{p}$ value $=0.0085$

variate in the adjusted model of the primary end point; however, it was not statistically significant when modeled as two linear splines $-<26 \mathrm{ml} / \mathrm{min} / 1.73 \mathrm{~m}^{2}(\mathrm{p}=0.246)$ and $\geq 26 \mathrm{ml} / \mathrm{min} / 1.73 \mathrm{~m}^{2}(\mathrm{p}=0.444)-$ and therefore was not included.

\section{Discussion}

As a part of conduct of the trial, subjects underwent treatment with epoetin-alfa to reach a steady-state level of drug effect according to the protocol-specified $\mathrm{Hb}$ target at 4 months. Those who incurred primary end points, tended to have the dose at 4 months maintained, while those without events had the dose reduced to achieve the protocol-determined $\mathrm{Hb}$ target over the ensuing months of the trial. When ignoring the randomization allocation and treatment target, we found those with the lowest epoetin-alfa dose exposure $(<5,164$ units/week) had reduced risks of primary events even if the $\mathrm{Hb}$ achieved was $>12.7 \mathrm{~g} / \mathrm{dl}$. Conversely, in the highest epoetin-alfa tertile and the lowest $\mathrm{Hb}$ achieved (11\% of the entire population), there was a nearly 2 -fold hazard for the composite outcome. The achieved $\mathrm{Hb}$ groupings revealed that there was a greater frequency of baseline heart failure in the 
highest tertile of $\mathrm{Hb}$ at 4 months, and this imbalance may have explained the association between the higher $\mathrm{Hb}$ target and heart failure hospitalization in the primary results of the CHOIR [3]. Thus, a higher Hb target did not place a subject at a higher risk of cardiovascular events unless the dose of epoetin-alfa was advanced in the middle or higher tertiles of $\mathrm{Hb}$ achieved. At the time of events, subjects were receiving higher doses of epoetin-alfa compared to those without events given similar per-patient time periods. These data suggest that the dose used at steady state (4 months) and the long-term maintenance doses of ESA used are the real determinants of epoetinalfa-associated cardiovascular events and not the $\mathrm{Hb}$ target or the $\mathrm{Hb}$ achieved [20]. Furthermore, there appeared to be a dose-related cardiovascular toxicity associated with epoetin-alfa above a cumulative median dose of 166,000 units (average $>10,095$ units/week, see Results text). Previous analyses from the CHOIR and other similar trials have concluded that resistance to ESA was the determinant of increased cardiovascular risk $[8,13,14]$. Our analysis is the first to examine the dose of epoetinalfa per week out to the time of first event or censure and clearly suggests that resistance to the drug which is typically determined within the first few weeks to months cannot explain the associations observed over the course of maintenance therapy. Those who were maintained on high doses of epoetin-alfa clearly had elevated risks irrespective of resistance or $\mathrm{Hb}$ response. We believe that if ESA resistance plays a role confounding cardiovascular risk (confounding by indication), then it is only in the highest dosing tertile and the lowest $\mathrm{Hb}$ response which represents approximately $11 \%$ of the population.

Similar to the CHOIR trial, the Normal Hematocrit study demonstrated an overall relative risk of 1.28, 95\% confidence interval $0.92-1.78$, favoring the low-hematocrit group [19]. However, subjects achieving a higher hematocrit with lower doses had a lower mortality rate than subjects in the lower-hematocrit group [19]. Higher $\mathrm{Hb}$ values alone in both the Normal Hematocrit study and CHOIR were not associated with worse outcomes if the epoetin-alfa dose was not escalated. Rather, lower $\mathrm{Hb}$ achieved values appeared to be associated with higher mortality even with lower doses of epoetin-alfa.

Epoetin-alfa requirements are variable among anemic patients [23]. This variability has been attributed to multiple etiologies, including iron deficiency, infection, and inflammation. Hyporesponsiveness to epoetin-alfa requires higher doses which currently do not have upper bounds found in clinical practice [24]. We observed approximately 143 -fold and 50-fold variations in the week- ly and cumulative doses of study drug received, creating an opportunity for considerable biologic variance in subjects. This could have led to an off-target cardiovascular adverse effect of epoetin-alpha.

Higher doses of epoetin-alpha have been demonstrated to be an independent predictor of mortality in the United States Renal Data System of dialysis patients [25]. Across all hematocrit categories, significant direct relationships between dose and mortality have been observed. The steepest increases in risk were found above the 72.5 th dose percentile, corresponding to 18,800 29,300 units/week, which are considerably greater than the $>10,095$ units/week which we found as the average dose received in the highest risk group over 378 days of treatment. Thamer et al. [26] found that the business model of a dialysis unit was strongly related to the average epoetin-alfa dose utilized. Among the 6 large chain facilities with a similar patient case mix, the average dose of epoetin ranged from 17,832 units/week at nonprofit facilities to 24,986 units/week at for-profit facilities. Compared with nonprofit facilities, for-profit facilities increased epoetin doses 3 -fold for patients with hematocrit levels of less than $33 \%(\mathrm{Hb}<11.0 \mathrm{~g} / \mathrm{dl})$.

Mechanisms by which high doses of epoetin-alfa may be associated with a greater risk of adverse outcomes remain unclear. Erythropoietin receptors have been demonstrated on human endothelial cells and multiple other sites [27, 28]. Additionally, receptors have been found on tumor cells suggesting potential roles of erythropoietin as a proliferative agent in vascular and myocardial tissue [29-31]. We recognize that large doses and perhaps increased frequency of doses both contributed to the cumulative dose exposure at 4 months. However, continued high-intensity doses appeared to be more common in those who incurred cardiovascular events. Thus, it is possible that not only the dose, but also the stimulation intensity over time has an adverse effect on the erythropoietin receptor that results in cardiovascular toxicity.

Our study has all the limitations of secondary analyses from clinical trials. Landmark analyses minimize biases created by differential dropout of subjects and intervening events between the time of randomization and the inception time for the outcome measurement. However, the impact of later $\mathrm{Hb}$ levels and doses received after the landmark time cannot be examined using this methodology. $\mathrm{Hb}$ target, actual $\mathrm{Hb}$ achieved, and epoetin-alfa dose are closely related in the CHOIR study due to the design of the trial. The dose utilized was a consequence of failure to respond, so by definition represents confounding by indication. However, the multivariable analysis clearly 
identified the weekly dose and not $\mathrm{Hb}$ as a determinant of cardiovascular events. While our findings are congruent with those concerning epoetin-alfa from the Normal Hematocrit Study, they may not apply to other ESAs such as darbepoetin. A similar analysis such as the one presented in this paper is urgently needed from the TREAT trial to confirm our findings. Such an analysis could have an important impact on prescribing physicians and predialysis chronic kidney disease patients receiving this ESA.

Our data partially support current US Food and Drug Administration guidance, for more conservative dosing of ESA in patients with chronic kidney disease. These recommendations now stress individualizing therapy for each patient and using the lowest possible ESA dose required to reduce the need for transfusions. These recommendations, however, still feature reduced $\mathrm{Hb}$ targets which in our view are irrelevant if the ESA dose is kept sufficiently low. In fact, our data suggest in those receiving low doses that normalization of $\mathrm{Hb}$ was not harmful [32]. We anticipate those with the highest $\mathrm{Hb}$ achieved had a quality-of-life benefit without the penalty of adverse cardiovascular outcomes. In summary, our analysis does not support restricted $\mathrm{Hb}$ targets for predialysis chronic kidney disease patients with anemia, rather a low dose ESA approach with monitoring and the final low maintenance dose continued even when there is a significant $\mathrm{Hb}$ response into the normal range $(>12.0 \mathrm{~g} / \mathrm{dl}$ for women and $>13.0 \mathrm{~g} / \mathrm{dl}$ for men).

\section{Acknowledgments}

The original CHOIR trial was supported by Ortho Biotech Clinical Affairs and Johnson \& Johnson Pharmaceutical Research and Development, both subsidiaries of Johnson \& Johnson. This analysis was supported by Duke Clinical Research Institute.

\section{Disclosure Statement}

Dr. McCullough has no relevant disclosures.

Dr. Barnhart reports receiving consulting fees and grant support from Ortho Biotech Clinical Affairs.

Dr. Szczech reports receiving consulting fees from Ortho Biotech Clinical Affairs, Nabi Pharmaceuticals, Gilead, Fresenius Medical Care, Kureha, Affymax and Acologix, lecture fees from Nabi Biopharmaceuticals, Fresenius Medical Care, GlaxoSmithKline, Gilead, Genzyme, Abbott, Amgen and Ortho Biotech, and grant support from Ortho Biotech Clinical Affairs, GlaxoSmithKline, Pfizer and Genzyme.

Dr. Inrig reports support by NIH grant K23 HL092297, lecture fees from Mitsubishi-Tanabe and Sanofi-Genzyme, and research grants and contracts from Genzyme, Reata and Keryx.

Dr. Reddan reports receiving consulting fees from Ortho Biotech Clinical Affairs and Shire Pharmaceuticals, lecture fees from Amgen, Novartis, Pfizer, AstraZeneca and General Electric, and grant support from Ortho Biotech Clinical Affairs, Amgen and Novartis.

Ms. Sapp has no disclosures.

Dr. Califf reports having received research grants and contracts from Merck, Novartis Pharmaceutical, Shering Plough, and Scios Pharma. He also reports receiving consulting fees from Avalere Health, Bayer, Biogen Idec, Brandeis University, Bristol Myers Squibb/Sanofi, Eli Lilly, Five Prime, Heart.org/Conceptis, Kowa Research Institute, Nitrox LLC, Sanofi-Aventis, and Vertex.

Dr. Patel reports support by NIH grant K23DK075929 and investigator-initiated research support from Abbott Laboratories.

Dr. Singh reports receiving consulting fees from Ortho Biotech Clinical Affairs, Amgen, Roche, Merck (Germany), Abbott, Watson and Horizon Blue Cross Blue Shield, and lecture fees from Ortho Biotech Clinical Affairs, Roche, Amgen, Abbott, Watson, Scios, Pfizer and Genzyme, serving on advisory boards for Ortho Biotech Clinical Affairs, Roche, Acologix, Watson, Advanced Magnetics and Amgen, and receiving grant support from Ortho Biotech Clinical Affairs, Dialysis Clinic, Roche, Baxter, Johnson \& Johnson, Amgen, Watson and Aspreva.

No other potential conflict of interest relevant to this article was reported.

\section{References}

1 Eschbach JW, Abdulhadi MH, Browne JK, Delano BG, Downing MR, Egrie JC, Evans RW, Friedman EA, Graber SE, Haley NR, et al: Recombinant human erythropoietin in anemic patients with end-stage renal disease. Results of a phase III multicenter clinical trial. Ann Intern Med 1989;111:992-1000.

-2 Bohlius J, Wilson J, Seidenfeld J, Piper M, Schwarzer G, Sandercock J, Trelle S, Weingart O, Bayliss S, Djulbegovic B, Bennett CL, Langensiepen S, Hyde C, Engert A: Recombinant human erythropoietins and cancer patients: updated meta-analysis of 57 studies including 9,353 patients. J Natl Cancer Inst 2006;98: 708-714.

-3 Singh AK, Szczech L, Tang KL, Barnhart H, Sapp S, Wolfson M, Reddan D: Correction of anemia with epoetin alfa in chronic kidney disease. N Engl J Med 2006;355:2085-2098.

4 Drueke TB, Locatelli F, Clyne N, Eckardt KU, Macdougall IC, Tsakiris D, Burger HU, Scherhag A: Normalization of hemoglobin level in patients with chronic kidney disease and anemia. N Engl J Med 2006;355:2071-2084.
5 Besarab A, Goodkin DA, Nissenson AR: The Normal Hematocrit Study - follow-up. N Engl J Med 2008;358:433-434.

-6 Pfeffer MA, Burdmann EA, Chen CY, Cooper ME, de Zeeuw D, Eckardt KU, Feyzi JM, Ivanovich $\mathrm{P}$, Kewalramani R, Levey AS, Lewis EF, McGill JB, McMurray JJ, Parfrey P, Parving $\mathrm{HH}$, Remuzzi $\mathrm{G}$, Singh AK, Solomon SD, Toto R, TREAT Investigators: A trial of darbepoetin alfa in type 2 diabetes and chronic kidney disease. N Engl J Med 2009;361:20192032. 
7 Skali H, Parving HH, Parfrey PS, Burdmann EA, Lewis EF, Ivanovich P, Keithi-Reddy SR, McGill JB, McMurray JJ, Singh AK, Solomon $\mathrm{SD}$, Uno $\mathrm{H}$, Pfeffer MA, TREAT Investigators: Stroke in patients with type 2 diabetes mellitus, chronic kidney disease, and anemia treated with darbepoetin alfa: the Trial to Reduce Cardiovascular Events with Aranesp Therapy (TREAT) experience. Circulation 2011;124:2903-2908.

-8 Szczech LA, Barnhart HX, Sapp S, Felker GM, Hernandez A, Reddan D, Califf RM, Inrig JK, Patel UD, Singh AK: A secondary analysis of the CHOIR trial shows that comorbid conditions differentially affect outcomes during anemia treatment. Kidney Int 2010;77:239246.

-9 McMurray JJ, Uno H, Jarolim P, Desai AS, de Zeeuw D, Eckardt KU, Ivanovich P, Levey AS, Lewis EF, McGill JB, Parfrey P, Parving HH, Toto RM, Solomon SD, Pfeffer MA: Predictors of fatal and nonfatal cardiovascular events in patients with type 2 diabetes mellitus, chronic kidney disease, and anemia: an analysis of the Trial to Reduce Cardiovascular Events with Aranesp (darbepoetin-alfa) Therapy (TREAT). Am Heart J 2011;162: 748-755.

10 Locatelli F, Aljama P, Canaud B, Covic A, De Francisco A, Macdougall IC, Wiecek A, Vanholder R, Anaemia Working Group of European Renal Best Practice (ERBP): Target haemoglobin to aim for with erythropoiesisstimulating agents: a position statement by ERBP following publication of the Trial to Reduce Cardiovascular Events with Aranesp Therapy (TREAT) study. Nephrol Dial Transplant 2010;25:2846-2850.

11 Locatelli F, Del Vecchio L: An expert opinion on the current treatment of anemia in patients with kidney disease. Expert Opin Pharmacother 2012;13:495-503.

12 McCullough PA, Lepor NE: The deadly triangle of anemia, renal insufficiency, and cardiovascular disease: implications for prognosis and treatment. Rev Cardiovasc Med 2005; 6:1-10.

$\checkmark 13$ Solomon SD, Uno H, Lewis EF, Eckardt KU, Lin J, Burdmann EA, de Zeeuw D, Ivanovich P, Levey AS, Parfrey P, Remuzzi G, Singh AK,
Toto R, Huang F, Rossert J, McMurray JJ, Pfeffer MA, Trial to Reduce Cardiovascular Events with Aranesp Therapy (TREAT) Investigators: Erythropoietic response and outcomes in kidney disease and type 2 diabetes. N Engl J Med 2010;363:1146-1155.

14 Winkelmayer WC: What caused excess strokes in patients randomized to darbepoetin in the Trial to Reduce Cardiovascular Events with Aranesp Therapy (TREAT)? No smoking gun. Circulation 2011;124:28052808.

15 Elliott J, Mishler D, Agarwal R: Hyporesponsiveness to erythropoietin: causes and management. Adv Chronic Kidney Dis 2009;16: 94-100.

16 Mallick S, Rafiroiu A, Kanthety R, Iqbal S, Malik R, Rahman M: Factors predicting erythropoietin resistance among maintenance hemodialysis patients. Blood Purif 2012;33:238-244.

17 Abiose AK, Aronow WS, Moreno H Jr, Nair CK, Blaschke TF, Hoffman BB: Increased vascular alpha1-adrenergic sensitivity in patients with renal failure: receiving recombinant erythropoeitin. Am J Ther 2007;14:427-434.

18 Raine AE: Hypertension, blood viscosity, and cardiovascular morbidity in renal failure: implications of erythropoietin therapy. Lancet 1988;1:97-100.

19 Besarab A, Bolton WK, Browne JK, Egrie JC, Nissenson AR, Okamoto DM, Schwab SJ, Goodkin DA: The effects of normal as compared with low hematocrit values in patients with cardiac disease who are receiving hemodialysis and epoetin. N Engl J Med 1998;339: 584-590.

20 Szczech LA, Barnhart HX, Inrig JK, Reddan DN, Sapp S, Califf RM, Patel UD, Singh AK: Secondary analysis of the CHOIR trial epoetin-alpha dose and achieved hemoglobin outcomes. Kidney Int 2008;74:791-798.

21 Singh AK, Szczech L, Tang KL, Barnhart H, Sapp S, Wolfson M, Reddan D: Correction of anemia with epoetin alfa in chronic kidney disease. N Engl J Med 2006;355:2085-2098.

-22 Levey AS, Coresh J, Greene T, Stevens LA, Zhang YL, Hendriksen S, Kusek JW, Van Lente F: Using standardized serum creatinine values in the modification of diet in renal dis- ease study equation for estimating glomerular filtration rate. Ann Intern Med 2006;145: 247-254.

23 Eschbach JW: Anemia management in chronic kidney disease: role of factors affecting epoetin responsiveness. J Am Soc Nephrol 2002; 13:1412-1414.

24 Coladonato JA, Frankenfield DL, Reddan DN, Klassen PS, Szczech LA, Johnson CA, Owen WF Jr: Trends in anemia management among US hemodialysis patients. J Am Soc Nephrol 2002;13:1288-1295.

25 Zhang Y, Thamer M, Stefanik K, Kaufman J, Cotter DJ: Epoetin requirements predict mortality in hemodialysis patients. Am J Kidney Dis 2004;44:866-876.

26 Thamer M, Zhang Y, Kaufman J, Cotter D, Dong F, Hernán MA: Dialysis facility ownership and epoetin dosing in patients receiving hemodialysis. JAMA 2007;297:1667-1674.

-27 Anagnostou A, Liu Z, Steiner M, Chin K, Lee ES, Kessimian N, Noguchi CT: Erythropoietin receptor mRNA expression in human endothelial cells. Proc Natl Acad Sci USA 1994; 91:3974-3978.

28 Juul SE, Yachnis AT, Christensen RD: Tissue distribution of erythropoietin and erythropoietin receptor in the developing human fetus. Early Hum Dev 1998;52:235-249.

29 Leo C, Horn LC, Rauscher C, Hentschel B, Liebmann A, Hildebrandt G, Hockel M: Expression of erythropoietin and erythropoietin receptor in cervical cancer and relationship to survival, hypoxia, and apoptosis. Clin Cancer Res 2006;12:6894-6900.

- 30 Mohyeldin A, Lu H, Dalgard C, Lai SY, Cohen N, Acs G, Verma A: Erythropoietin signaling promotes invasiveness of human head and neck squamous cell carcinoma. Neoplasia 2005;7:537-543.

31 Ribatti D, Marzullo A, Gentile A, Longo V, Nico B, Vacca A, Dammacco F: Erythropoietin/erythropoietin-receptor system is involved in angiogenesis in human hepatocellular carcinoma. Histopathology 2007;50: 591-596.

32 Manns BJ, Tonelli M: The new FDA labeling for ESA - implications for patients and providers. Clin J Am Soc Nephrol 2012;7:348353. 